\title{
Designing an Antenna System That Can Perform Conditional RF to DC Harnessing To Generate Electricity
}

\author{
Raghunandan G. Kalibhat ${ }^{1}$, Arun L. Kakhandki ${ }^{2}$, Ramakrishna S. ${ }^{3}$ \\ Dr.Priyatamkumar ${ }^{4}$ \\ ${ }^{I}$ U.G. Student (Dept. of Electronics and Communication Engineering, B.V.Bhoomraddi College of Engineering \\ and Technology, Hubli ,India) \\ ${ }^{2}$ Associate Professor (Dept. of Electronics and Communication Engineering, B.V.Bhoomraddi College of \\ Engineering and Technology, Hubli ,India) \\ ${ }^{3}$ Assistant Professor (Dept. of Electronics and Communication Engineering, B.V.Bhoomraddi College of \\ Engineering and Technology, Hubli ,India) \\ ${ }^{4}$ Professor (Dept. of Electronics and Communication Engineering, B.V.Bhoomraddi College of Engineering and \\ Technology, Hubli ,India)
}

\begin{abstract}
Electromagnetic energy or RF energy will play a pivotal role in wireless technology and wireless communication in the impending future. The paper proposes a concept for a patch antenna based system that can harness $R F$ energy upon triggering and can convert the harnessed $R F$ to DC from the radio frequency of 1 $\mathrm{GHz}$ to $3 \mathrm{GHz}$, the design frequency is $2.4 \mathrm{GHz}$. The patch antenna system contains a high gain patch antenna along with a wireless communicating module and a conversion circuit. The return loss of the antenna is approximately 27.1 dB. The power gain is $30.1 \mathrm{dBm}$.The converter circuit is designed in), Multi-Sim to get an output voltage of around $5 \mathrm{~V}$ that can be used to power a mobile-device or maybe stored in a battery. The triggering part is done with the help of a T-mote which is simulated in a network simulator, Cooja. The patch antenna is simulated in High Frequency Structural Simulator.
\end{abstract}

Keywords: Cooja, HFSS (High Frequency Structural Simulator), Multi-Sim, Patch antenna

\section{Introduction}

The wireless technology has dominated its predecessor in many aspects like the ease of use, long range accessibility. Also with the ever-growing developments it also made affordable for a common person. Wireless technology uses radio waves to transmit information without cables or wiring. Wireless operations permit services, such as long-range communications, that are impossible or impractical to implement with the use of wires. Common methods of achieving wireless communications include the use of other electromagnetic wireless technologies, such as light, magnetic, or electric fields or the use of sound. One of the major and recent developments in the same is the use of wireless charging for mobile applications. Mobile giant Samsung has come out with the concept of electricity and magnetism to develop a wireless charging system. This system consists of an inductance pad. Placing the mobile phone on the pad will charge it automatically. In electromagnetic theory, it is classified as a near-field activity. The charging pad so developed makes use of the concept of mutual inductance to accomplish the same. A current flowing through the pad will give rise to a magnetic flux. This magnetic flux is then linked to the mobile phone by mutual inductance. The Lenz's law comes into picture here. The mobile phone there-by tries to prevent the cause for this flux by generating a current. This current is then controlled and used for charging the phone. Hence, the near-field activity makes use of magnetism. On the contrary, far-field activity makes use of electromagnetic waves especially the microwaves for its applications. These electromagnetic waves can be easily generated using an oscillator or some other source and then be transmitted with the help of an antenna. At the receiving end, a receiver antenna captures these waves and then passes it for the further processing. Since these waves carry both electrical and magnetic energy, both or either of these can be extracted and used for different applications.

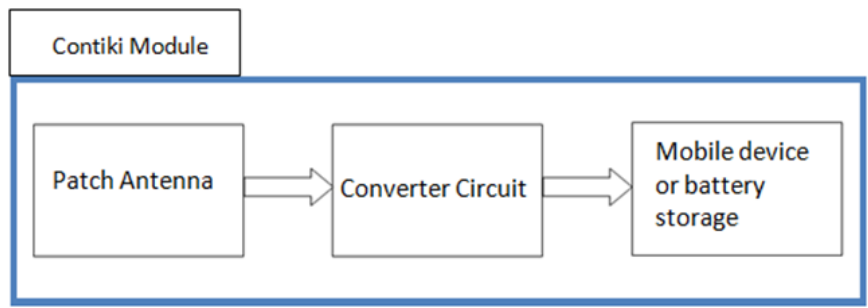

Fig 1. Patch Antenna based system 


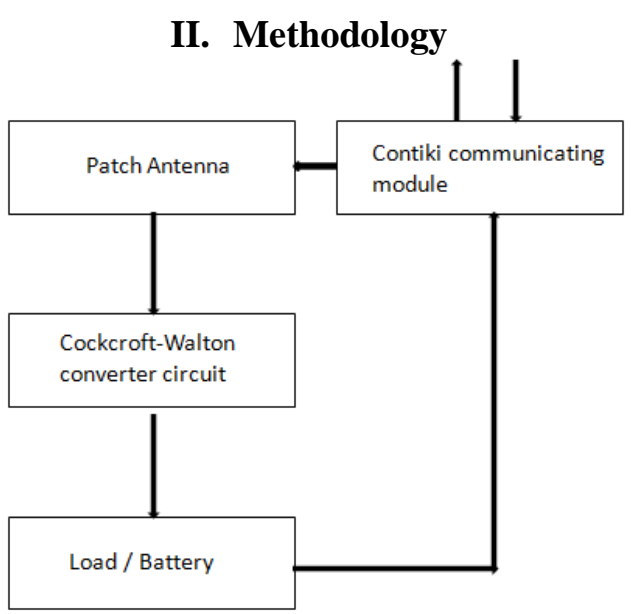

Fig 2. Flowchart

\subsection{Antenna Modeling}

The antenna modeled and simulated with the help of simulation software by Ansys. The antenna designed is a patch antenna with the operation range of $1-3 \mathrm{GHz}$. The desired frequency of operation of the patch antenna simulated in High Frequency Structural Software is $2.4 \mathrm{GHz}$. The substrate used for the patch antenna is FR4 or Fire Resistant epoxy which has a very good mechanical strength also with an efficiency of $99.6 \%$. It also helps in size reduction. FR4 provides a relatively high bandwidth when compared to other materials used for substrate like Roger-4350, Benzocyclobutane etc. The specification of the FR4 substrate used in this concept is as shown in the table.

Table 1. Antenna material specification

\begin{tabular}{|l|l|}
\hline \multicolumn{1}{|c|}{ Description } & Value \\
\hline Material Used & FR4 epoxy \\
\hline Frequency of operation & $2.4 \mathrm{GHz}$ \\
\hline Width & $38.03 \mathrm{~mm}$ \\
\hline Height & $29.46 \mathrm{~mm}$ \\
\hline Dielectric Constant & 4.4 \\
\hline
\end{tabular}

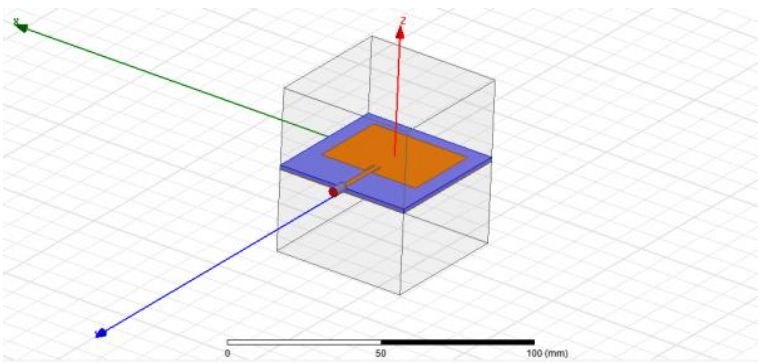

Fig 3. Antenna modeling using HFSS

\subsection{Circuit Simulation}

The circuit simulation is done using the Multi-Sim software by National Instruments. The RF-DC converter circuit is a Cockcroft-Walton multipier circuit. The peculiarity of this circuit is that it not only converts the AC to DC, but also elevates the level of output obtained. Since the operation involves dealing with the micro-waves traditional diodes cannot be used. Schottky diodes 10BQ015 along with diodes 1BH62 here act as the rectifiers. The first part of the circuit elevates the AC level which is rectified by the second part of the circuit. The RF wave received by the antenna is converted to voltage. Here, for the frequency of $2.4 \mathrm{GHz}$ an alternating voltage of $7.19 \mathrm{~V}$ is given as input to the converter circuit. The first part of the circuit elevates it to $9.617 \mathrm{~V}$ which is later brought down $5.4 \mathrm{~V}$.

Table 2. Descripton of circuit components

\begin{tabular}{|l|l|c|}
\hline Description & \multicolumn{1}{|c|}{ Notation } & Ratings \\
\hline Capacitors & C1,C2,C3,C4,C5 & $1 \mathrm{nF}, 10 \mathrm{~V}$ \\
\hline Diodes(Fast-recovery) & D6,D7,D8,D9 & $100 \mathrm{~V}, 1 \mathrm{~A}$ \\
\hline Schottky diodes & D2,D3,D4,D5 & $3 \mathrm{GHz}$ \\
\hline Resistors & R2,R3,R4 & $1 \mathrm{~K}$ ohm and 2.5K ohm \\
\hline
\end{tabular}




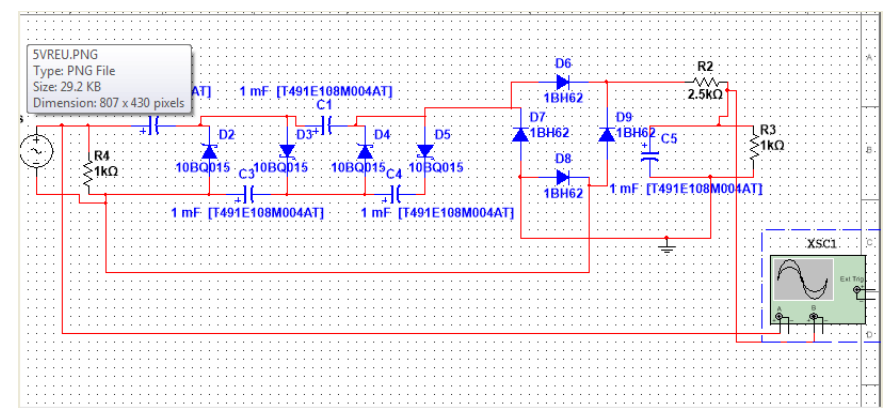

Fig 4. Cockroft-Walton circuit simulation using Multi-Sim

\subsection{Contiki Module}

The contiki module is used for the purpose of communication between the transmitter and receiver systems. Contiki 2.7 OS is used here.The module consists of a T-mote. The T-mote is simulated in the Cooja network simulator and the results obtained are shown in the figure. Communication between the two motes is simulated under two conditions once when they are in range and when they are out of range and is shown below.
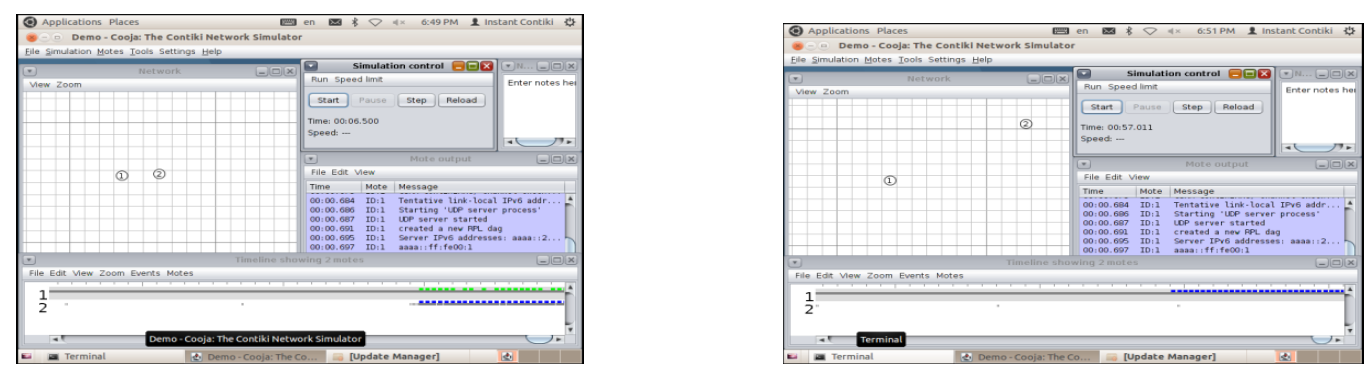

Fig 5. Mote simulation in Cooja

\section{Procedure}

The design of patch antenna was done on a substrate with the dimensions $38 \mathrm{~mm}$ X $29 \mathrm{~mm}$. The antenna is a high gain antenna with a frequency of operation from $1-3 \mathrm{GHz}$. The power gain curve shows that the antenna has a gain of $30.15 \mathrm{dBm}$. This is equivalent to an rms voltage of $7.19 \mathrm{~V}, 2.4 \mathrm{GHz}$. This is given as the input to the Cockcroft-Walton circuit. The circuit in turn provides an output to $9.6 \mathrm{~V}$ that is brought down to $5.4 \mathrm{~V}$. Now, the working of the system is as follows. Consider a transmitter and receiver along with their corresponding circuits respectively. The transmitter circuit can be an oscillator that can generate desired frequencies. The research makes an important assumption of the Contiki-communicating module being present in the transmitter antenna as well as the receptor antenna. To begin with whenever there is a need for charges/ energy for an end device say mobile-phone, it sends a request to the receptor Contiki T-mote. This module in turn sends a request to the transmitter Contiki T-mote. It allows for the transmission of the microwave signals generated by the oscillator. An actuator can be used at the receptor antenna to allow it to capture the microwave signals. The transmitting antenna must be aimed properly to receive more power. The received power is then harnessed in to DC for powering the load.

Table 3. Abbrevations and Acronyms

\begin{tabular}{|c|c|}
\hline DC & Direct Current \\
\hline RF & Radio Frequency \\
\hline HFSS & High Frequency Structural Software \\
\hline
\end{tabular}

Unit

- $\mathrm{dBm}$ - power gain of antenna

- $\mathrm{GHz}-$ frequency

\section{Results}

The working frequency of the antenna is as shown in the figure along with its power gain. Return loss can be imagined as the ratio of power incident on the antenna to the power lost as a result of lossy nature (due to different types of antenna apertures that play role in heating the antenna, dissipating the power).The graph of return loss in $\mathrm{dB}$ vs frequency is plotted and is shown. 


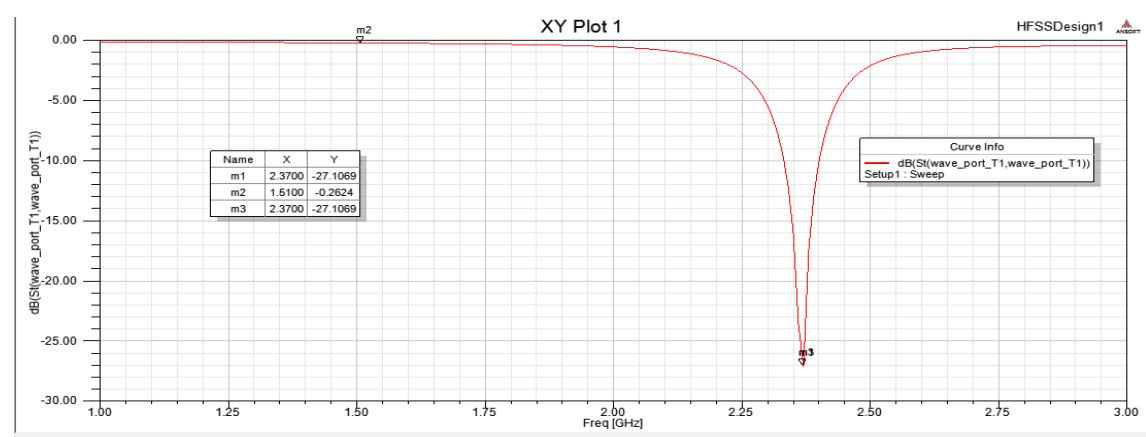

Fig 6. Return loss of the patch antenna

The graph shows that the antenna has a return loss of $27.1 \mathrm{~dB}$ at the designed frequency of $2.4 \mathrm{GHz}$. The powergain graph shown depicts a maximum power of $30.15 \mathrm{dBm}$. Power gain is the graph of peak gain in $\mathrm{dBm}$ vs theta.

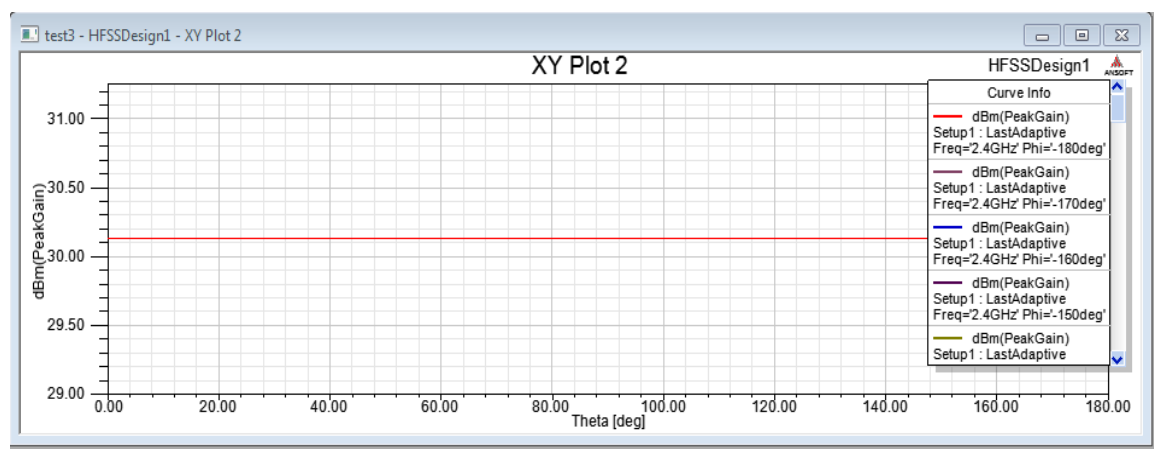

Fig 7. Power gain of the antenna

As explained earlier the converter circuit has two stages. The first stage elevates the signal. The second stage rectifies it. The output is shown at the end of the first stage and then the second stage respectively.

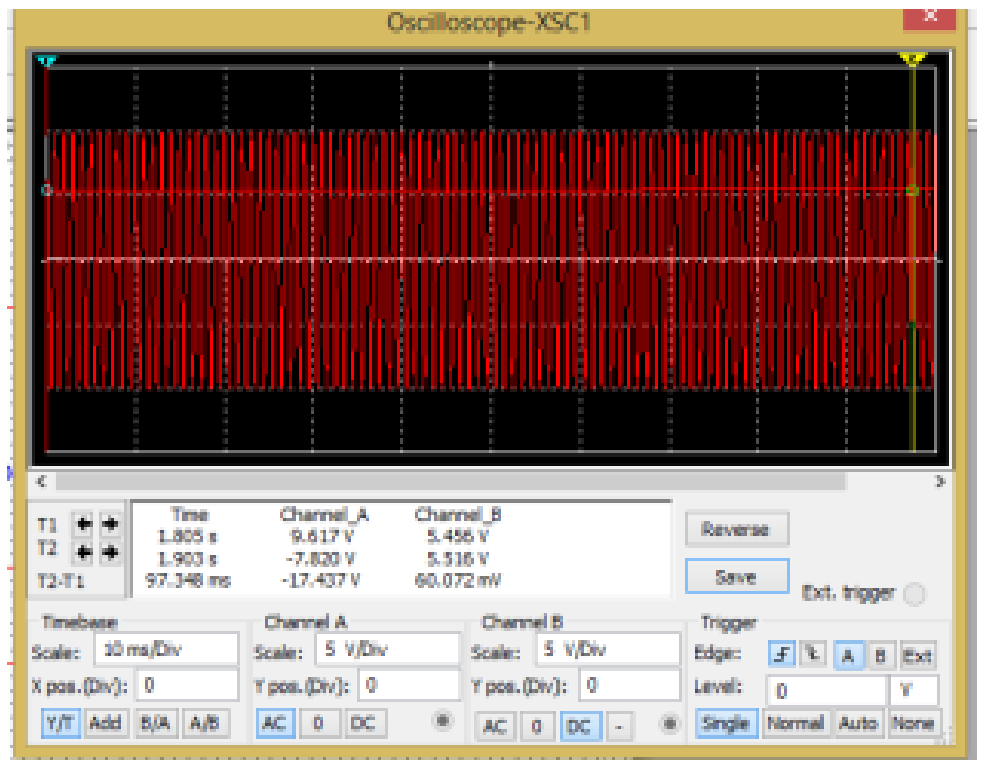

Fig 8. Output of the cockroft-walton converter circuit

Since the transmission of the microwave signals can be only triggered upon the interception of messages by the Contiki T-mote, placing of the T-motes also plays an important role as they communicate over the radio. The following snippets show how the efficiency varies with the distance. In the first snippet the motes are in range and the distance is around $10 \mathrm{~m}$. In the second snippet the motes are out of range and the distance is around $30 \mathrm{~m}$. 

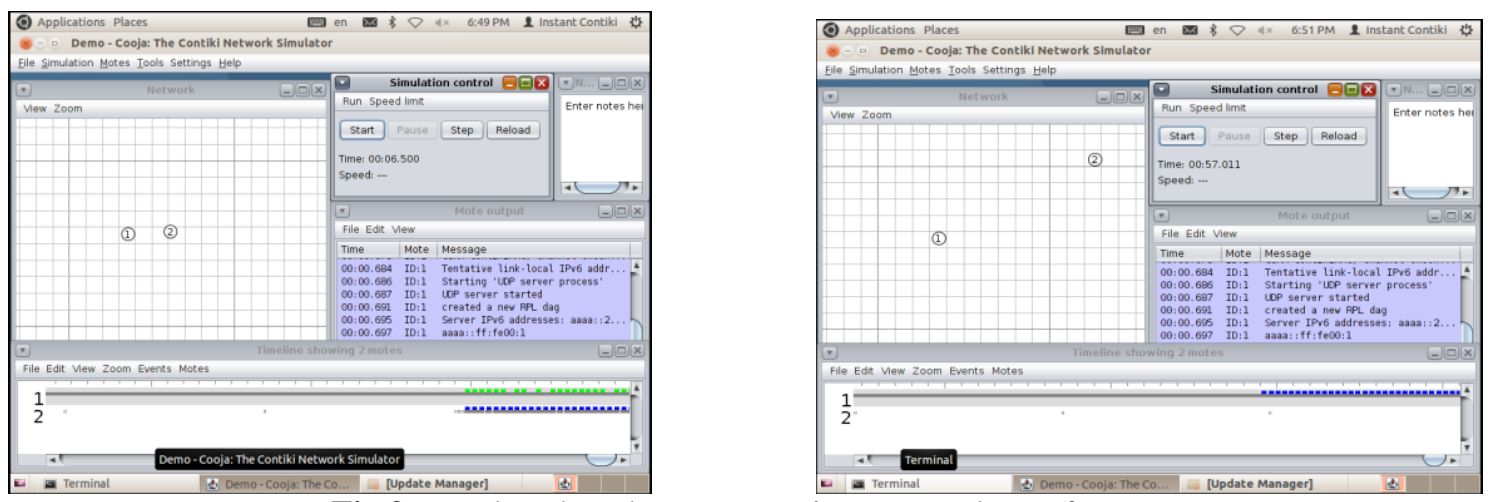

Fig 9. Results when the motes are in range and out of range

\section{Conclusion}

The present research makes use of a substrate of dimensions $38 \mathrm{~mm}$ X $29 \mathrm{~mm}$. This dimension can be reduced or be more optimised to make the system more portable. Further the communication between the transmitter and receiver system can be made more efficient by using alternative mechanisms. This in turn may increase the working distance enhancing the efficincy of reception also.More focus can be levied on communication between the motes and the load in case if the system is purely used for charging application to achieve complete automation.

\section{References}

[1]. Balanis, Constantine A. Antenna theory: analysis and design. John Wiley \& Sons, 2016

[2]. $\quad$ Fatthi Alsager, Ahmed. "Design and Analysis of Microstrip Patch Antenna Arrays." (2011).

[3]. Prasad, K. D., and Deepak Handa. Antenna and wave propagation. Satya Prakashan, 2003.

[4]. Kraus, John D., and Ronald J. Marhefka. "Antenna for all applications." Upper Saddle River, NJ: McGraw Hill (2002).

[5]. Reddy, M. Venkateswara, K. Sai Hemanth, and CH Venkat Mohan. "Microwave Power Transmission-A Next Generation Power Transmission System." IOSR Journal of Electrical and Electronics Engineering (IOSRJEEE), e-ISSN (2013): 2278-1676.

[6]. Gabrillo, L. J., M. G. Galesand, and J. A. Hora. "Enhanced RF to DC converter with LC resonant circuit." IOP Conference Series: Materials Science and Engineering. Vol. 79. No. 1. IOP Publishing, 2015.

[7]. Mane, Mrs Punita, S. A. Patil, and Mr PC Dhanawade. "Comparative Study of Microstrip Antenna for Different Subsrtate Material at Different Frequencies."

[8]. Bouchouicha, D., et al. "Ambient RF energy harvesting." International Conference on Renewable Energies and Power Quality. 2010.

[9]. Wang, Jiang, Liang Dong, and Yuzhuo Fu. "Modeling of UHF voltage multiplier for radio-triggered wake-up circuits." International Journal of Circuit Theory and Applications 39.11 (2011): 1189-1197.

[10]. Tentzeris, Manos M., and Yoshihiro Kawahara. "Novel energy harvesting technologies for ICT applications." Applications and the Internet, 2008. SAINT 2008. International Symposium on. IEEE, 2008.

[11]. Sample, Alanson, and Joshua R. Smith. "Experimental results with two wireless power transfer systems." Radio and Wireless Symposium, 2009. RWS'09. IEEE. IEEE, 2009.

[12]. Yan, Han, et al. "An integration scheme for RF power harvesting." Proc. STW Annual Workshop on Semiconductor Advances for Future Electronics and Sensors. 2005.

[13]. Harrist, Daniel W. Wireless battery charging system using radio frequency energy harvesting. Diss. University of Pittsburgh, 2004.

[14]. Ganapathy, Shrikanth, et al. "Dynamic fine-grain body biasing of caches with latency and leakage 3T1D-based monitors." Computer Design (ICCD), 2011 IEEE 29th International Conference on. IEEE, 2011.

[15]. Brown, W., J. Mims, and N. Heenan. "An experimental microwave-powered helicopter." 1958 IRE International Convention Record. Vol. 13. IEEE, 1966.

[16]. Tesla, Nikola. "The transmission of electrical energy without wires as a means for furthering peace." Electrical World and Engineer 7 (1905): 21.

[17]. Yoo, Tae-Whan. Experimental and theoretical study on 35 GHz RF-to-DC power conversion receiver for millimeter-wave beamed power transmission. Diss. Texas A\&M University, 1993. 